La Revue

des Droits

de l'Homme

\section{La Revue des droits de l'homme}

Revue du Centre de recherches et d'études sur les droits fondamentaux

Actualités Droits-Libertés | 2017

\title{
La sortie du territoire chinois d'un demandeur d'asile sous sa véritable identité constitue-t-elle un motif de refus d'une demande d'asile?
}

Droit d'asile (Principe du contradictoire)

Daniel Arthur Laprès

\section{OpenEdition}

Journals

Édition électronique

URL : http://journals.openedition.org/revdh/3349

DOI : $10.4000 /$ revdh.3349

ISSN : 2264-119X

Éditeur

Centre de recherches et d'études sur les droits fondamentaux

Référence électronique

Daniel Arthur Laprès, « La sortie du territoire chinois d'un demandeur d'asile sous sa véritable identité constitue-t-elle un motif de refus d'une demande d'asile ? ", La Revue des droits de l'homme [En ligne], Actualités Droits-Libertés, mis en ligne le 06 novembre 2017, consulté le 04 mai 2019. URL : http:// journals.openedition.org/revdh/3349; DOI : 10.4000/revdh.3349

Ce document a été généré automatiquement le 4 mai 2019.

Tous droits réservés 


\section{La sortie du territoire chinois d'un demandeur d'asile sous sa véritable identité constitue-t-elle un motif de refus d'une demande d'asile?}

Droit d'asile (Principe du contradictoire)

Daniel Arthur Laprès

\section{Introduction}

1 Dans une série de décisions rendues récemment, l'office Français de Protection des Réfugiés et Apatrides (OFPRA) et la Cour Nationale du Droit d'Asile (CNDA) ont justifié le rejet des demandes d'admission au statut de réfugié présentées par des ressortissants chinois parce que ces derniers ont réussi à se faire délivrer des passeports chinois en utilisant leurs véritables identités et qu'ils ont pu passer les contrôles aux aéroports chinois en présentant ces passeports.

2 Au yeux de l'OFPRA et de la CNDA, ces faits signifient que les personnes concernées n'étaient pas recherchées par la police chinoise, moyennant quoi leurs allégations de persécutions aux mains des autorités chinoises seraient nécessairement fausses.

Ainsi, ces décisions partent du présupposé selon lequel le Gouvernement chinois aurait mis en place un système de surveillance informatisé d'une telle efficacité que toute personne recherchée pour les comportements ayant donné lieu aux persécutions alléguées par les demandeurs d'asile aurait été repérée soit lors du traitement de sa demande de passeport, moyennant quoi sa demande aurait été rejetée, soit lors de son passage aux contrôles frontaliers à l'aéroport d'embarquement en Chine. A en suivre le raisonnement de la CNDA et de l'OFPRA, les ressortissants Chinois en cause auraient été empêchés de quitter le territoire chinois s'ils avaient réellement été inquiétés par la police dans les conditions alléguées pour justifier leurs craintes de persécution. 
4 Le premier juin 2016, la CNDA a ainsi rejeté les recours d'un couple de chinois au motif, notamment, que leurs « craintes (de persécutions) apparaissent peu crédibles dès lors qu'(ils ont) obtenu la délivrance officielle d'un passeport ... et qu'(ils ont) quitté légalement la République Populaire de Chine alors qu'(ils) affirment avoir été poursuivi(s) et placé(s) sous surveillance ${ }^{1} »$.

Par ordonnance du 6 juillet $2017^{2}$, la CNDA, validant le raisonnement de l'OFPRA, a rejeté le recours d'une chinoise qui avait quitté son pays avec son propre passeport en citant, sans plus de précisions, un rapport de la Commission de l'Immigration et du Statut de Réfugié du Canada (CISR) publié le 6 mars 2014³.

Dans une décision rendue le 21 février 2017, l'OFPRA a retenu comme seul motif, pour refuser la demande, le fait que la demanderesse a pu obtenir l'aval du Bureau des Entrées et des Sorties en Chine pour quitter le territoire et que celle-ci a pu passer les contrôles d'identité mis en œuvre à l'aéroport, éléments qui « démontrent une complaisance des autorités ». Dès lors, selon l'OFPRA, «la surveillance dont (la demanderesse) aurait fait l'objet après sa libération et les recherches menées à son encontre ne peuvent être tenues pour établies. . . Par conséquent, l'office ne peut conclure au bien-fondé des craintes exprimées par l'intéressée ${ }^{4} »$. Dans sa décision, l'OFPRA mentionne avoir consulté un rapport interne intitulé «DIDR, Interdictions de sortie du territoire, législation et contrôles effectués aux postes frontières, 7 octobre 2016 \%.

7 De manière similaire, dans deux décisions rendues le 18 avril 2017, l'OFPRA a considéré «peu vraisemblable que les autorités chinoises aient pu délivrer un passeport régulier à une personne recherchée, depuis le 1er octobre 2014, du fait de son appartenance à une église souterraine » et «les motifs de craintes ne sauraient, dès lors, être considérées comme établis ${ }^{5} »$.

8 Enfin, par une décision rendue le 28 avril 2017, l'OFPRA a rejeté la demande sous prétexte que l'obtention par la demanderesse d'un passeport et son passage sans incident lors des contrôles policiers à l'aéroport chinois excluaient « de tenir les faits allégués pour établis (et) de regarder comme fondées les craintes de persécutions exprimées ${ }^{6} »$.

9 L'auteur souhaite présenter une critique de ces décisions, en soulignant au premier chef que les procédures permettant l'invocation des rapports officiels canadiens sans les verser au dossier violent le principe du contradictoire (1).

En second lieu, l'auteur souhaite souligner que la fiabilité supposée des rapports de l'administration canadienne cités par la CNDA et l'OFPRA pour fonder leurs appréciations est contestable (2) ${ }^{7}$.

11 Enfin, l'auteur procédera à une analyse de la jurisprudence des tribunaux canadiens qui, sur cette question, contredisent largement les rapports de l'administration canadienne (3).

\section{$1^{\circ} /$ Une violation du principe du contradictoire}

12 Dans aucune des affaires ci-dessus citées, les demandeurs n'ont été informés, avant la réception des rejets de leurs demandes par l'OFPRA ou de leurs recours par la CNDA, des sources sur lesquelles ces derniers se sont fondés pour parvenir à la conclusion qu'il est interdit de délivrer un passeport chinois à des personnes ayant les profils des demandeurs d'asile concernés et que de telles personnes seraient empêchées par la police 
chinoise de quitter le territoire chinois lors de leur passage aux contrôles aéroportuaires. L'absence de communication aux demandeurs d'asile des sources sur lesquelles la CNDA et l'OFPRA entendaient fonder leurs conclusions, en les privant ainsi d'une possibilité d'en commenter la pertinence et la fiabilité, constitue une violation du principe du contradictoire tant au regard des règles en droit interne que de celles applicables en droit de l'Union Européenne.

\section{A)Les règles de procédure applicables en droit interne devant I'OFPRA et la CNDA} d'information générale librement accessibles au public dont elle doit alors indiquer l'origine dans sa décision ${ }^{8} »$. Force est de constater que l'une des décisions de la CNDA commentées, soit celle rendue le 7 juillet 2017, respecte cette règle puisqu'elle comporte une mention de la source justifiant la conclusion que la sortie de la Chine par la demanderesse avec son propre passeport compromettait la crédibilité de ses allégations de persécutions. respecter le principe du contradictoire en communiquant au requérant «les éléments d'information susceptibles de confirmer ou d'infirmer des circonstances de fait propres au demandeur d'asile ou spécifiques à son récit ».

Plus particulièrement, le Conseil d'Etat a jugé que

«Considérant qu'en se fondant pour rejeter la requête de M. C...sur des 'sources documentaires internationalement reconnues' dont l'examen aurait permis de démentir la véracité d'un fait individuel précis allégué par le requérant et justifiant sa demande d'asile, 'sources' dont aucune pièce du dossier qui était soumis à la cour ne permettait d'identifier l'origine, la nature et le contenu, et sans que la décision indique à quelles pièces du dossier cette qualification pouvait éventuellement s'appliquer, la Cour nationale du droit d'asile, faisant ainsi reposer sa décision sur des éléments d'information susceptibles de confirmer ou d'infirmer des circonstances de fait propres au demandeur d'asile ou spécifiques à son récit qui n'avaient pas été soumises au contradictoire, l'a entachée d'irrégularité. »

Plus récemment, le Conseil d'Etat a réitéré cette règle en les termes suivants :

«[...] il incombe (à la CNDA), dans la mise en œuvre de ses pouvoirs d'instruction, de veiller au respect des droits des parties, d'assurer l'égalité des armes entre elles et de garantir, selon les modalités propres à chacun d'entre eux, les secrets protégés par la loi ; que le caractère contradictoire de la procédure fait en principe obstacle à ce que le juge se fonde sur des pièces produites au cours de l'instance qui n'auraient pas été préalablement communiquées à chacune des parties?

Le constat par la CNDA que des demandeurs d'asile aient pu obtenir des passeports chinois et passer les contrôles policiers aux aéroports chinois sans être arrêtés correspond à notre sens a à des « faits propres à leurs récits » mentionnés par l'arrêt précité du Conseil d'Etat. Aussi, l'inférence par la CNDA que ces événements n'auraient pas pu se produire si les intéressés étaient poursuivis par la police chinoise dépend logiquement d'autres éléments. Ainsi, le fait qu'une personne soit recherchée en Chine équivaut dans certains cas à une raison pour l'obliger ou, en tout cas, pour lui permettre en tant qu'indésirable de quitter le territoire. Donc, la CNDA n'a pu énoncer que les requérants n'auraient pu obtenir des passeports chinois et quitter le territoire chinois 
sous leur identité réelle sans se fonder nécessairement sur d'autres éléments, qui n'ayant pas été versés au dossier n'ont pas été soumis au contradictoire.

Les éléments complémentaires nécessaires peuvent relever du droit, soit une réglementation chinoise prévoyant le refus de délivrance de passeports et l'interdiction de quitter le territoire et/ou des faits tels que des constats par des observateurs de refus de délivrance de passeports et d'empêchements de quitter le territoire. De telles réglementations ou observations visant des personnes ayant le profil des demandeurs d'asile chinois constituent des "éléments d'information susceptibles de confirmer ou d'infirmer des circonstances de fait propres au demandeur d'asile ou spécifiques à leur récit ».

19 Ainsi, dans l'hypothèse où la CNDA apprécierait une demande d'asile d'un chinois ayant franchi la frontière chinoise avec son propre passeport à la lumière de «sources documentaires internationalement reconnues ", elle devrait assurer que ces sources sont versées au dossier en identifiant les "origine, nature et contenu» des informations concernées et en fournissant au requérant une possibilité de les commenter.

Cette obligation de transparence incombe aussi à l'OFPRA en application du Code de l'entrée et du séjour des étrangers et du droit d'asile (CESEDA) .dont l'article L 723-4 impose à l'OFPRA l'obligation « d'évaluer, en coopération avec le demandeur, les éléments pertinents de la demande ».

Dans un arrêt ${ }^{10}$ interprétant l'obligation de coopération imposée par l'article 4 de la Directive 2004/83/CE ${ }^{11}$, la Cour de Justice de l'Union Européenne (CJUE) a jugé que cette obligation de "coopération", qui s'impose dès le premier niveau d'examen d'une demande d'admission au statut de réfugié, signifie que :

«[...]concrètement, si, pour quelque raison que ce soit, les éléments fournis par le demandeur d'une protection internationale ne sont pas complets, actuels ou pertinents, il est nécessaire que l'état membre concerné coopère activement, à ce stade de la procédure, avec le demandeur pour permettre la réunion de l'ensemble des éléments de nature à étayer la demande "

Puisque la CJUE impose une telle obligation de coopération proactive, il est raisonnable de prévoir qu'elle sanctionnerait l'omission de remettre au demandeur d'asile des documents dont dispose l'OFPRA et sur lesquels il entend fonder un rejet de la demande.

\section{B)Les règles de procédure applicables en droit de l'Union Européenne}

Le 23 juin 2013, le Parlement Européen et le Conseil ont adopté la Directive 2013/32/UE relative à des procédures communes pour l'octroi et le retrait de la protection internationale (la Directive Européenne) dont l'article 12 impose une obligation de transparence dans les procédures afférant à l'examen des demandes d'asile

Selon l'article 12 de la Directive Européenne pré-citée ${ }^{12}$ :

1. En ce qui concerne les procédures prévues au chapitre III (Procédures en première instance), les États membres veillent à ce que tous les demandeurs bénéficient des garanties suivantes:

d) ils ont accès et, le cas échéant, leurs conseils juridiques ou autres conseillers ont accès, conformément à l'article 23, paragraphe 1 , aux informations visées à l'article 10 , paragraphe 3 , point b) ... lorsque l'autorité responsable de la détermination a 
tenu compte de ces informations pour prendre une décision relative à leur demande. » communiquer aux demandeurs d'asile chinois contre lesquels ces institutions entendent prononcer des décisions qui leur sont défavorables toutes les sources sur lesquelles ils se fondent pour juger que leur sortie du territoire chinois avec leurs propres passeports compromet la crédibilité de leurs allégations de persécutions dans leur pays. Commissariat des Nations Unies pour les refugiés ainsi que les organisations Commissariat des Nations Unies pour les réfugiés ainsi que les organisations internationales compétentes en matière de droits de l'homme, sur la situation générale existant dans les pays d'origine des demandeurs et, le cas échéant, dans les pays par lesquels les demandeurs ont transité [...]

Donc, lorsque l'OFPRA se réfère à des sources identifiées au point b) ci-dessus, elle a l'obligation de communiquer aux demandeurs d'asile des exemplaires des sources dont elle tient compte pour rendre sa décision, et ceci avant de prendre ladite décision.

En outre, cette obligation découle également d'un principe général du droit de l'Union Européenne

Bien que l'article 41(2) de la Charte des droits fondamentaux qui garantit aux administrés l'accès aux dossiers qui les concernent, ait été jugé opposable aux seules institutions, organes et organismes de l'Union ${ }^{13}$, ce droit est reconnu en tant que principe général du droit de l'Union Européenne ${ }^{14}$ et il est donc susceptible d'être invoqué par des demandeurs d'asile dans le cadre de l'examen de leurs dossiers par l'OFPRA.

Dans son arrêt M.G. et R.N. ${ }^{15}$, la CJUE a jugé que :

«[...] selon une jurisprudence constante, les droits de la défense, qui comportent le droit d'être entendu et le droit d'accès au dossier, figurent au nombre des droits fondamentaux faisant partie intégrante de l'ordre juridique de l'Union et consacrés par la Charte. Il est vrai également que le respect de ces droits s'impose même lorsque la réglementation applicable ne prévoit pas expressément une telle formalité. »

Plus récemment encore, dans l'affaire Mukarubega ${ }^{16}$, la CJUE a qualifié «le respect des droits de la défense » comme " principe fondamental du droit de l'Union » et "principe général du droit de l'Union». Dans cette affaire, il était certes question du droit d'être entendu qui :

[...]garantit à toute personne la possibilité de faire connaître, de manière utile et effective, son point de vue au cours de la procédure administrative et avant l'adoption de toute décision susceptible d'affecter de manière défavorable ses intérêts ${ }^{17}$.

Le droit d'accès au dossier étant une autre facette des droits de la défense et étant intégré par exemple à l'article 41 (2) de la Charte des droits fondamentaux dans le même alinéa et au même niveau que le droit d'être entendu, cette jurisprudence de la CJUE garantit in fine aux demandeurs d'asile le droit d'avoir accès à toutes les pièces sur lesquelles l'OFPRA entend se fonder pour rejeter de leurs demandes.

En résumé, en droit, la CNDA et l'OFPRA devraient, avant de rendre leurs décisions, 


\section{$2^{\circ} /$ La portée des rapports administratifs canadiens cités par la CNDA et par l'OFPRA}

Dans les affaires citées ci-dessus, ni la CNDA ni l'OFPRA n'ont fait état d'éléments démontrant de manière convaincante que les personnes ayant été inquiétées par les autorités en Chine dans les conditions typiquement alléguées par les demandeurs d'asile originaires de ce pays se voient soit refuser leur demande de passeport, soit interdire de quitter le territoire chinois par les policiers aux aéroports.

Dans l'une des affaires commentées, la CNDA cite en soutien de ce motif un rapport de la CISR du Canada publié le 6 mars $2014^{18}$ reprenant en cela une citation dans la décision de l'OFPRA ayant fait l'objet du recours ${ }^{19}$. Dans une autre affaire, l'OFPRA a cité ce même rapport dans sa décision ${ }^{20}$.

Dans seulement l'une de ces affaires, l'OFPRA a joint à sa décision transmise au demandeur un exemplaire du rapport concerné sur lequel le motif était fondé, s'agissant du rapport interne de l'OFPRA référencé « DIDR du 7 octobre $2016^{21}$ ».

Ce rapport interne de l'OFPRA cite l'article 12 de la loi de la République Populaire de Chine sur la gestion des entrées et sorties ${ }^{22}$ disposant que « sont interdites de quitter le territoire les personnes impliquées dans une procédure pénale en tant qu'accusé ou suspect ».

Pour le reste, le rapport interne de l'OFPRA renvoie essentiellement à des rapports établis par la CISR publiés respectivement le 20 février $2014^{23}$, le 6 mars, $2014^{24}$ et le 7 mars $2014^{25}$

40 Enfin sur le site de la $\mathrm{CNDA}^{26}$ sont mentionnés encore d'autres rapports canadiens, mais ayant été publiés en $2007^{27}$ et $2009^{28}$, ces derniers ne sont plus d'actualité.

Aucun des rapports canadiens ne comporte d'affirmation que des "craintes (de persécutions) apparaissent peu crédibles dès lors qu'(un demandeur d'asile) a obtenu la délivrance officielle d'un passeport . . et qu'(il) a quitté légalement la République Populaire de Chine alors qu'(il) affirme avoir été poursuivi et placé sous surveillance ", selon la formule adoptée par la CNDA ${ }^{29}$.

Chacun des rapports canadiens précise au surplus que ses auteurs ne prétendent pas « apporter des preuves concluantes quant au fondement d'une demande d'asile ${ }^{30}$ ».

Pourtant, selon l'OFPRA, dans sa décision $\mathrm{N}^{\circ}$ 15-06-02098, les rapports canadiens démontrent que

«[...] les mesures de contrôle aéroportuaires en Chine sont particulièrement strictes, en particulier pour les dissidents politiques et religieux et pour toute personne susceptible de compromettre la sécurité de l'Etat [...]

les agents de sécurité ont accès à la base de données en ligne, Policenet, laquelle contient les noms des citoyens déclarés coupables de crimes ou bien recherchés par les autorités . .. cette base est établie par le Bureau de la Sécurité Publique et si une personne fait l'objet de recherches, le balayage électronique de son passeport signale sa situation et s'ensuit une détention immédiate pour interrogatoire et une interdiction de quitter le territoire. » Mais, dans son rapport interne cité ci-dessus, l'OFPRA résume des déclarations de tiers comprises dans ces rapports canadiens : 

justifier leur conclusion qu'une personne ayant été inquiétée dans les circonstances typiquement alléguées par les demandeurs d'asile chinois pour cause de persécutions à motif religieux serait nécessairement interdite de quitter le territoire chinois. un Guide Jurisprudentiel ${ }^{38}$ incitant au rejet des demandes d'asile de chinois ayant quitté leur pays par les aéroports en présentant leur propre passeport.

«Le secrétaire général de la Dui Hua Foundation [...] a affirmé que les autorités saisissent les pièces d'identité et les titres de voyage appartenant aux citoyens chinois qui sont maintenus en détention criminelle. Le secrétaire général a précisé qu'il est difficile de savoir si un citoyen qui fait l'objet d'une enquête, mais qui n'a pas été officiellement détenu, se fait saisir ses pièces d'identité et ses titres de voyage ${ }^{31}$. »

Les rapports canadiens citent des sources diverses déclarant avoir observé une gamme de circonstances justifiant l'inclusion d'une personne dans les systèmes informatiques de suivi des citoyens, mais aucune de ces sources n'allègue que les personnes interpellées pour des infractions à la réglementation administrative afférant à la religion en font partie (distinguant ainsi les interventions policières visant la répression d'infractions pénales de celles régulant les infractions administratives).

Un des rapports canadiens cite l'agence de presse chinoise Xinhua annonçant l'existence d'un système unifié de casiers judiciaires ainsi que de systèmes d'information « dans les catégories des enquêtes criminelles intégrées, des fugitifs, des empreintes digitales prélevées sur les lieux de crime, des voitures volées, des narcotiques et de l'antiterrorisme ${ }^{32} »$.

Ce reportage de Xinhua est commenté de la manière suivante par les auteurs du rapport canadien:

«Parmi les sources qu'elle a consultées dans les délais fixés, la Direction des recherches n'a trouvé aucun renseignement supplémentaire ni aucun renseignement allant dans le même sens que ceux présentés ci-dessus ${ }^{33}$.»

Dans le même rapport canadien, le magazine The Economist est cité comme ayant constaté que des données officielles sur le fonctionnement des deux systèmes de censure en ligne gérés par l'Etat en Chine, la Grande Muraille pare-feu et le Bouclier d'or, sont inaccessibles « pour des raisons évidentes ${ }^{34}$ ».

Le rapport cite également au même paragraphe un spécialiste de l'Université de Toronto qui, dans un article publié par le New York Times, écrivait que les chercheurs occidentaux savent « remarquablement peu ${ }^{35}$ » sur l'étendue de la surveillance étatique en Chine. Et ce constat est validé par la Laogai Research Foundation ${ }^{36}$.

Enfin, selon l'organisation non gouvernementale Freedom House, cité dans le rapport canadien, «le filtrage est hétérogène et souvent inégal, compte tenu du moment, de la technologie et de la région géographique ${ }^{37} »$.

En résumé, les sources canadiennes citées par l'OFPRA et la CNDA ne semblent pas Certes, la Section des Appels des Réfugiés (SAR) de la CISR a publié le 30 novembre 2016

Néanmoins, ce Guide n'a pas encore été invoqué par l'OFPRA ou la CNDA.

En tout état de cause, le Guide Jurisprudentiel reprend les éléments soutenant les rapports canadiens antérieurs et ses auteurs retiennent que

«[...]le gouvernement chinois dispose d'un réseau informatique national, connu sous le nom du projet Bouclier d'or et que le PSB a accès à une base de données nationale de la police qui contient de l'information sur les criminels fugitifs, sur les passeports ainsi que 
sur les entrées et les sorties. Le Bouclier d'or intègre de nombreux mécanismes de suivi et de contrôle, dont la technologie de surveillance de la reconnaissance faciale ${ }^{39}$. "

Pour la préparation du Guide, d'autres sources ont été ajoutées au dossier. Ainsi, il est précisé qu' " [...]un représentant de l'ambassade du Canada a affirmé que, avant le départ, une personne peut avoir à présenter son passeport quatre fois à l'intérieur de l'aéroport. Le représentant de l'ambassade a aussi affirmé que le passeport est balayé deux fois : au comptoir d'enregistrement de la ligne aérienne et au comptoir des départs du service de l'immigration. La même source a signalé que le billet d'avion du passager était balayé à l'entrée de la [traduction] « zone de transit sécurisée » et à la porte d'embarquement de la ligne aérienne $e^{40}$.

D'autre part, la SAR a consulté l'Association du transport aérien international (la section Sûreté aérienne et facilitation) qui a signalé qu'«à sa connaissance, la procédure de départ aux aéroports de la Chine comportait trois étapes: 1) l'enregistrement; 2) le contrôle au service d'immigration; et 3) le contrôle de sécurité et que, à ces trois points, les passagers sont tenus de présenter leur passeport ${ }^{41}$. »

Néanmoins ni les services diplomatiques canadiens ni l'IATA n'indiquent que les compagnies aériennes ont accès au réseau Bouclier d'or ni que leurs contrôles ont comme finalité autre chose que la vérification de la conformité des renseignements sur le passeport du voyageur avec ceux fournis pour réserver son billet d'avion.

Soulignons qu'en outre, le Guide Jurisprudentiel entreprend une spéculation concernant la vraisemblance d'échapper aux contrôles par la corruption :

"Selon la SAR, il est raisonnable de s'attendre à ce qu'une personne qui tente d'éviter l'arrestation et la détention en quittant le pays veuille savoir quels services pouvait fournir le passeur qu'il embauchait et quelles mesures seraient prises pour qu'il puisse quitter la Chine en toute sécurité. La SAR est d'avis qu'il n'est pas logique que l'appelant ait simplement mis sa vie entre les mains d'un passeur inconnu sans obtenir de renseignements détaillés sur le plan qui serait mis en œuvre pour quitter le pays ${ }^{42}$. »

Le Guide Jurisprudentiel sous-entend donc que le voyageur a dû exiger du passeur un grand nombre de précisions concernant les conditions de son passage aux points de contrôle qu'il devrait pouvoir décrire dans le cadre de sa demande d'asile. Et, faute de pouvoir fournir de tels détails lors de son entretien d'asile, les allégations des requérants selon lesquelles ils auraient corrompu des fonctionnaires à l'aéroport ne seraient pas crédibles.

Ce raisonnement nous paraît pour le moins fallacieux en ce qu'il est tout aussi plausible que le passeur ait tenu à garder secrètes les dispositions mises en œuvre et les contacts exploités, non seulement pour minimiser les risques de dénonciation, mais également afin de pouvoir réitérer les procédures pour d'autres personnes.

De plus, si l'on suppose - comme le font les auteurs du Guide Jurisprudentiel - que le passeur a accompagné le demandeur d'asile quand il s'est présenté aux contrôles aéroportuaires, le besoin de fournir des détails de son plan aux fins de rassurance est dissipé par la garantie que représente sa présence aux côtés de son " client ».

Selon le Guide Jurisprudentiel, l'allégation selon laquelle la police chinoise a continué à rechercher le demandeur d'asile depuis son arrivée au Canada «mine l'argument selon lequel son nom aurait été, en quelque sorte, supprimé du système $\mathrm{e}^{43} »$. Ainsi, les auteurs du Guide présupposent de manière contestable que la seule façon de passer les contrôles consisterait à effacer le nom du demandeur d'asile du réseau Bouclier d'Or. 
Constatons toutefois que les auteurs du rapport n'ont aucun moyen de prouver la conformité de cette supposition à la réalité. Aussi, tant qu'à spéculer, pourquoi ne pas envisager que le nom du voyageur ait été temporairement effacé du système informatique?

Les auteurs du Guide avancent certes une explication, affirmant qu' « Il est raisonnable de s'attendre à ce que l'utilisation de cet appareil fasse aussi l'objet d'une surveillance et que des systèmes redondants soient en place pour empêcher que le système soit compromis par une seule personne ${ }^{44} »$. Toutefois, une telle affirmation relève une fois de plus, selon nous, de la spéculation

71 En résumé, ni les sources canadiennes spécifiquement référencées par l'OFPRA et la CNDA, ni le Guide Jurisprudentiel ne comporte des arguments convaincants pour soutenir la conclusion que tout ressortissant chinois ayant été inquiété par les autorités à cause de la libre pratique de sa religion serait repéré lors des contrôles aéroportuaires de son identité et qu'il serait dès lors interdit de quitter le territoire, même en ayant eu recours à l'aide d'un passeur.

\section{$3^{\circ} /$ Les conséquences à tirer du fait qu'un demandeur d'asile chinois ait pu quitter le territoire chinois avec son propre passeport : apport de la jurisprudence canadienne.}

Bien que l'OFPRA et la CNDA n'aient cité que les rapports administratifs canadiens sur les questions en cause, il existe bien une jurisprudence méticuleusement raisonnée et qui est contraire à la ligne adoptée par les instances de la CISR.

Sous l'empire régime fédéral canadien, l'administration des demandes d'asile relève de la compétence du gouvernement national. Les demandes d'asile sont d'abord entendues par la Section de Protection (SPR) de la CISR ; elle les instruit et décide de les accepter ou non. Ses décisions sont généralement susceptibles de faire l'objet d'appels devant la SAR. Des recours contre les décisions de cette dernière peuvent être portés devant la Cour Fédérale, ensuite devant la Cour d'Appel Fédérale, et enfin devant la Cour Suprême du Canada.

74 Une série de six jugements de la Cour Fédérale ${ }^{45}$, dont la plus récente rendue le 10 août 2017 dans l'affaire Huang c Canada ${ }^{46}$ - et sous réserve de deux décisions divergentes rendues en $2015^{47}$ et $2016^{48}$ - ont énoncé qu'une SAR commet une erreur viciant sa décision lorsqu'elle rejette une demande d'asile d'un ressortissant chinois au motif d'un manque de crédibilité de ses allégations de crainte de persécution lorsque la décision est prise au motif que le requérant n'aurait pas pu quitter le territoire chinois en utilisant son passeport s'il avait été vraiment recherché par les autorités chinoises.

Dans l'affaire Huang, il incombait à la Cour Fédérale de juger si la SAR avait apprécié « raisonnablement» les allégations de fait du requérant. Cette exigence implique «l'existence dans le processus de décision de justification, de transparence et d'intelligibilité » moyennant quoi la Cour n'intervient que lorsque la décision critiquée se situe en dehors de la "gamme de résultats possibles, acceptables, susceptibles d'être défendus au regard des faits et du droit ${ }^{49}$ ». Dans cette affaire, le demandeur d'asile avait déclaré avoir engagé les services d'un passeur professionnel qui avait organisé son 
exfiltration avec son propre passeport en passant les contrôles frontaliers après avoir corrompu les fonctionnaires à l'aéroport ou autres services concernés.

Dans cette affaire, la SAR, en se fondant sur les mêmes informations que celles incluses dans les rapports administratifs canadiens ci-dessus référencés, avait conclu que ces allégations étaient dénuées de fondement. Par conséquent, la Cour a dû évaluer le caractère « raisonnable » du rejet par la SAR des allégations de fait de Monsieur Huang. La Cour a annulé la décision de la SAR en la lui renvoyant pour une nouvelle appréciation en application des principes fixés dans son arrêt, observant en l'espèce qu'en Chine, «Des fonctionnaires corrompus sont soudoyés afin d'aider des migrants chinois et font partie de groupes de passeurs qui disposent de connaissances concernant les systèmes de sécurité en vigueur ${ }^{50} »$.

La Cour canadienne a, dans sa décision, fait référence à un rapport australien constatant que « En Chine, il existe une corruption systématique et les fonctionnaires aux aéroports sont susceptibles d'être soudoyés ${ }^{51}$ » et que «les autorités en Chine n'appliquent pas toujours uniformément les règlements ${ }^{52} \%$. La Cour canadienne a également retenu l'existence de cas de chinois recherchés par la police qui ont réussi à passer les contrôles aux aéroports et à sortir du territoire chinois avec leur propre passeport ${ }^{53}$.

Enfin, la juridiction a souligné que rien ne contredit la possibilité d'éviter le fichage dans le système informatique en ne soudoyant qu'un seul fonctionnaire, d'autant que les chefs des réseaux de passeurs sont souvent des policiers ${ }^{54}$ et qu'il est concevable que les informations collectées au niveau local ne soient pas centralisées par l'administration chinoise..$^{55}$.

\section{Conclusion}

Il faut en premier lieu espérer que l'OFPRA et la CNDA renonceront à la pratique, devenant systématique, de se fonder sur des rapports provenant d'autorités étrangères pour rejeter des demandes d'asile sans même donner aux demandeurs la possibilité de les commenter avant la prise de décision.

Les rapports administratifs canadiens invoqués par l'OFPRA et la CNDA ne justifient pas la conclusion qui en est pourtant tirée qu'un ressortissant chinois recherché par la police chinoise ne pourrait ni obtenir un passeport ni passer les contrôles aéroportuaires avec ce passeport sans être repéré et interdit de quitter le territoire.

81 Surtout, alors qu'il existe une jurisprudence canadienne qui rejette la recommandation des rapports administratifs canadiens, cette jurisprudence n'a pas été citée par l'OFPRA ou par la CNDA, en tout cas dans les affaires ici en cause. Or, pour autant que ces autorités françaises estiment utile de se référer à des sources canadiennes, il y aurait lieu de privilégier les sources judiciaires, déterminantes sur les questions en cause, plutôt que les sources secondaires que sont les rapports administratifs.

82 Ensuite, dans les affaires ici évoquées, l'OFPRA et la CNDA ont considéré que les demandeurs chinois se disaient « recherchés » par la police, laquelle observation appelle plusieurs commentaires.

83 Premièrement, les demandeurs d'asile chinois en France prétendent avoir été « arrêtés " et/ou avoir été «surveillés » plus souvent qu'ils ne se disent avoir été «recherchés ». 
Dans certains cas, il y a éventuellement eu introduction de la notion de "recherché » au cours des interrogatoires, que ce soit par l'OFPRA ou par la CNDA.

Et même à supposer que le demandeur d'asile ait avancé qu'il était recherché par la police dans son pays, est-il vraisemblable qu'un chinois puisse obtenir de la police locale une information fiable sur le point de savoir s'il est « recherché »?

Cette différence entre les notions de "surveillance » ou « arrestation », d'une part, et de "recherche », d'autre part, est importante car la réglementation chinoise prévoit le fichage de personnes qui sont au moins "suspectées » de crimes, si ce n'est poursuivies devant un tribunal et même condamnées.

Or, il semble évident qu'une personne puisse avoir été "arrêtée » sans jamais être enregistrée dans le système informatique Bouclier d'Or, surtout quand elle n'est pas poursuivie devant les tribunaux. Aussi, les demandeurs d'asile chinois ne prétendent presque jamais avoir été poursuivis devant les tribunaux chinois.

En supposant qu'un demandeur d'asile prétende avoir été "arrêté » au sens de la loi chinoise ou interrogé en tant que "suspect » dans une affaire pénale, il serait pertinent de savoir si les obligations en droit chinois envers le « suspect » ont été respectées.

Par exemple, la police chinoise est censée informer un suspect qu'il peut faire intervenir un défenseur ${ }^{56}$. Mais, cette question n'a été posée ni par l'OFPRA ni par la CNDA dans les affaires ici traitées., moyennant quoi il leur était impossible de savoir si ladite arrestation ou interrogation en tant que suspect avait été effectuée dans un cadre légal Or, cette précision est importante puisqu'au cas où l'interrogatoire ou l'arrestation par la police chinoise ne serait pas régulière au sens de la loi chinoise afférant à la procédure pénale, il serait hasardeux de supposer son enregistrement dans le réseau Bouclier d'Or.

$\mathrm{Au}$ surplus, d'après l'article 26 de la circulaire afférant aux procédures à mettre en œuvre par les organes de sécurité publique dans le cadre de la gestion des affaires criminelles, la police est investie de la faculté (mais pas de l'obligation) d'exiger « en fonction des circonstances » qu'une personne libérée sous caution doive remettre son passeport ${ }^{57}$.

91 De plus, lorsque les demandeurs d'asile chinois disent avoir été «surveillés », il s'agit le plus souvent de surveillance par des voisins zélés ou par le réseau de télésurveillance installée plus ou moins intensément à travers le pays.

Or, rien dans la littérature n'accrédite l'idée qu'une personne ainsi « surveillée » doit être fichée dans le réseau Bouclier d'Or.

Enfin, pour ce qui concerne les demandeurs d'asile chinois en France qui invoquent le risque de persécutions en raison de leur libre pratique de la religion en Chine, ceux-ci se décrivent le plus souvent comme des adeptes d'Eglises clandestines forcés de pratiquer leur religion dans leurs domiciles, en violation du droit chinois. Ce comportement n'est pas en tant que tel sanctionné par la loi pénale chinoise, mais l'est plutôt en application de dispositions administratives. Donc, les personnes "arrêtées ", «interrogées » ou « suspectées » en relation avec de telles infractions ne seraient pas normalement incluses dans le réseau Bouclier d'Or, qui n'enregistre que les infractions à la législation pénale. Bien entendu, ceci n'empêche pas nécessairement la police de les menacer de poursuites pénales, ni des les maintenir en dehors de tout cadre légal dans des circonstances justifiant leurs craintes de persécutions à cause de leur foi ${ }^{58}$. 
En résumé, l'OFPRA et la CNDA ont tort de rejeter des demandes d'asile de chinois invoquant leurs craintes de persécutions en raison de leur religion pour le motif qu'ils ont pu quitter le territoire chinois sous leur propre identité et l'invocation des arguments critiquant cette motivation n'engendre pas nécessairement des contradictions susceptibles de compromettre leurs demandes pour d'autres motifs.

CNDA, 4ème chambre, 2ème section, Décision n 15030201, premier juin 2016, Madame W. ép. H., et CNDA, 4ème chambre, 2ème section, Décision n ${ }^{\circ}$ 15030200, premier juin 2016, M. $\mathrm{H}$.

CNDA, Décision $\mathrm{n}^{\circ}$ 17018419, 6 juillet 2017, Madame Z. Y.

OFPRA, Décision n 15-06-02098, 21 février 2017, Madame Z., T. .

OFPRA, Décision No 16-09-209999, 18 avril 2017, Monsieur C., et OFPRA, Décision $\mathrm{N}^{\circ}$ 16-09-209966, 18 avril 2017, Madame Z., ép. : C..

Les Lettres « Actualités Droits-Libertés » (ADL) du CREDOF (pour s'y abonner) sont accessibles sur le site de la Revue des Droits de l'Homme (RevDH) - Contact

\section{BIBLIOGRAPHIE}

OFPRA, Décision n 16-09-21211, 28 avril 2017, Madame D..

\section{NOTES}

1. CNDA, $4^{\text {ème }}$ chambre, $2^{\text {ème }}$ section, Décision $n^{\circ} 15030201$, premier juin 2016, Madame W. ép. H., et CNDA, $4^{\text {ème }}$ chambre, $2^{\text {ème }}$ section, Décision $n^{\circ} 15030200$, premier juin 2016, M. H.

2. CNDA, Décision $n^{\circ} 17018419,6$ juillet 2017, Madame Z. Y. La CNDA a tranché par ordonnance en application de l'article L 733-2 du Code de l'entrée et du séjour des étrangers et du droit d'asile (CESEDA) parce que le recours ne présentait aucun élément sérieux susceptible de remettre en cause la décision de l'OFPRA ( $\mathrm{N}^{\circ} 16-09-25694,31$ mars 2017).

3. CISR, "Chine: information sur les mesures de contrôle et de sécurité à la sortie dans les aéroports s'appliquant aux citoyens chinois qui se rendent à l'étranger, y compris la procédure aux postes de contrôle et le recours à la vérification informatisée de l'identité; la communication de renseignements aux agents dans les aéroports ", 6 mars 2014.

4. OFPRA, Décision n 15-06-02098, 21 février 2017, Madame Z., T..

5. OFPRA, Décision $\mathrm{N}^{\circ}$ 16-09-209999, 18 avril 2017, Monsieur C., et OFPRA, Décision $\mathrm{N}^{\circ}$ 16-09-209966, 18 avril 2017, Madame Z., ép. : C..

6. OFPRA, Décision n 16-09-21211, 28 avril 2017, Madame D.. 
7. Les décisions de l'OFPRA ici critiquées font l'objet de recours en annulation devant la CNDA qui sont en cours d'instruction.

8. CE,10ème et 9ème sous-sections réunies, $\mathrm{n}^{\circ}$ 328265, 22 octobre 2012.

9. $\mathrm{CE}, \mathrm{n}^{\circ} 349560$, le premier octobre 2014.

10. CJUE, première chambre, 22 novembre 2012, affaire M.M., C-277/11.

11. Cette Directive est entrée en vigueur le 20 octobre 2004 et l'article 4 a été transposé en l'article 723-4 du CESEDA.

12. La Directive devait être transposée en droit interne avant le 20 juillet 2015 (article 51 de la Directive). Il est indifférent de savoir si la loi de transposition de cette Directive (Loi $\mathrm{n}^{\circ}$ 2015-925 du 29 juillet 2015 relative à la réforme du droit d'asile) inclut une mesure correspondant audit article 12 puisque le délai de transposition étant expiré, ses exigences peuvent être invoquées par un demandeur d'asile pour contester un acte administratif le concernant (CE, Assemblée, 30 octobre 2009, $\mathrm{n}^{\circ}$ 298348).

13. CJUE, 22 novembre 2012, M.M., Aff. C-277/11 et CJUE, 5 novembre 2014, Mukarubega, Aff. C-166/13, paragraphe 44 CJUE, 17 juillet 2014, YS e. a., Aff. jointes C-141/12 et C-372/12, paragraphe 67.

14. CJUE, 22 novembre 2012, M.M., Aff. C-277/11, paragraphes 49, 81, 82, 88 et 93.

15. CJUE,10 septembre 2013, M.G. et R.N., Aff. C-383/13, paragraphe 32.

16. CJUE, 5 novembre 2014, Mukarubega, Aff. C-166/13, paragraphe 45.

17. CJUE, 5 novembre 2014, Mukarubega, Aff. C-166/13, paragraphe 46.

18. CNDA, nº 17018419, Madame Z., Y., 6 juillet 2017.

19. OFPRA, $n^{\circ}$ 16-0925694, Madame Z., $Y, 31$ mars 2017

20. OFPRA, n 1506-02098, Madame Z. ép. L., 21 février 2017.

21. OFPRA, Interdictions de sortie du territoire, 7 octobre 2016.

22. La Loi afférant aux entrées et aux sorties, adoptée à la 27ème session du Comité Permanent de la 11ème l'Assemblée Populaire Nationale (APN) le 30 juin 2012, qui est entrée en vigueur le premier juillet 2013.

23. «Chine : information indiquant si les autorités saisissent le passeport des personnes visées par des citations à comparaître en matière criminelle, par une enquête de la police ou par des accusations au criminel »

24. «Chine : information sur les mesures de contrôle et de sécurité à la sortie dans les aéroports s'appliquant aux citoyens chinois qui se rendent à l'étranger, y compris la procédure aux postes de contrôle et le recours à la vérification informatisée de l'identité; la communication de renseignements aux agents dans les aéroports, lequel rapport est invoqué par l'OFPRA et la CNDA dans une affaire citée ci-dessus qui a abouti au rejet de la demande d'une chinoise. »

25. «Chine : information sur les mesures de contrôle et de sécurité à la sortie dans les aéroports s'appliquant aux citoyens chinois qui se rendent à l'étranger, y compris la procédure aux postes de contrôle et le recours à la vérification informatisée de l'identité; la communication de renseignements aux agents dans les aéroports. ». Le rapport de l'OFPRA cite également un autre rapport de la CISR, mais il n'éclaire pas les questions ici discutées : « CISR, Chine : information sur les exigences et la marche à suivre pour obtenir des certificats de sortie, y compris sur l'autorité de délivrance, le délai de traitement et les motifs de refus prévus dans la loi sur la gestion des entrées et des sorties adoptée en 2012, 2 février 2015»

26. A la date de cet article, la page en question en fonctionne plus puisque le lien intitulé «Dossiers pays» est désormais affiché un écran indiquant que «Cette rubrique est en cours de mise à jour ..." (http://www.cnda.fr/Ressources-juridiques-et-geopolitiques/Les-dossiers-pays et http://www.cnda.fr/cartables/). Mais, la page telle qu'antérieurement affichée peut être consultée au http://www.lapres.net/cndachinedossier.pdf. 
27. CISR, Information indiquant si le Bureau de la sécurité publique (BSP) saisit des cartes d'identité ou des passeports au domicile des personnes qu'il compte arrêter; circonstances dans lesquelles des pièces d'identité sont saisies et si un reçu est délivré, 13 juin 2007.

28. CISR, Information indiquant si le Bureau de la sécurité publique (BSP) possède un réseau informatique national permettant l'échange de renseignements; information sur la nature et l'importance des communications entre les bureaux du BSP à l'échelle nationale; information indiquant si les aéroports internationaux chinois ont accès à un réseau informatique de la police, 2 juillet 2009

29. CNDA, 4ème chambre, 2ème section, Décision n 15030201, premier juin 2016, Madame W. ép. H., et CNDA, 4ème chambre, 2ème section, Décision n 15030200, premier juin 2016, M. H.

30. Par exemple, CISR, Chine : information sur le projet Bouclier d'or du Bureau de la sécurité publique (Public Security Bureau), y compris sa mise en œuvre et son efficacité; information sur Policenet, y compris les zones d'activité; information sur le niveau et l'efficacité de l'échange de renseignements entre les autorités, 7 mars 2014, section 5 .

31. OFPRA, Interdictions de sortie du territoire, op.cit.page 3.

32. CISR, Chine : information sur le projet Bouclier d'or du Bureau de la sécurité publique (Public Security Bureau), y compris sa mise en cuvre et son efficacité; information sur Policenet, y compris les zones d'activité; information sur le niveau et l'efficacité de l'échange de renseignements entre les autorités, 7 mars 2014, section 4.

33. Ibid.

34. Ibid., section 5 .

35. Ibid.

36. Ibid.

37. Ibid., section 2 .

38. CISR, Guide Jurisprudentiel, SAR, $n^{\circ}$ TB6-11632, 30 novembre 2016, http://www.irb-cisr.gc.ca/ Fra/BoaCom/references/pol/jurisprud/Pages/TB6-11632.aspx.

39. Ibid., paragraphe 13 .

40. Ibid., paragraphe 15 .

41. Ibid., paragraphe 17.

42. Ibid., paragraphe 23.

43. Ibid., paragraphe 36 .

44. Ibid., paragraphe 28.

45. Zhang v Canada (Citizenship and Immigration), 2008 FC 533, paragraphes 5, 9, 10 ; Sun v Canada (Citizenship and Immigration), 2015 FC 387, paragraphes 13, 26 ; Ren v Canada (Citizenship and Immigration), 2015 FC 1402, paragraphes 16 ; Yang v Canada, (Citizenship and Immigration), 2016 FC 543, paragraphes 12-14 ; Yao v Canada (Citizenship and Immigration), 2016 FC 927, paragraphe 9.

46. Huang v Canada (Citizenship and Immigration), N 2017 FC 762, 10 août 2017, http:// decisions.fct-cf.gc.ca/fc-cf/decisions/en/item/233588/index.do.

47. Ma v Canada (Citizenship and Immigration), 2015 FC 838, 9 juillet 2015. Dans cette affaire, la SPR, la SAR et les juges de la Cour Fédérale ont estimé que le récit du demandeur d'asile à propos de son recours aux services d'un passeur manquait en crédibilité car trop « confus » et variant ", http://decisions.fct-cf.gc.ca/fc-cf/decisions/en/item/111046/index.do.

48. Sui v. Canada (Citizenship and Immigration), 2016 FC 406, 14 avril 2016. Dans cette affaire, le demandeur d'asile a prétendu qu'il était sorti trois fois du territoire et ces allégations ont été jugées comme peu crédibles, http://decisions.fct-cf.gc.ca/fc-cf/decisions/en/item/143841/ index.do.

49. Huang v Canada (Citizenship and Immigration), N 2017 FC 762, 10 août 2017, http:// decisions.fct-cf.gc.ca/fc-cf/decisions/en/item/233588/index.do, paragraphe 25.

50. Ibid., paragraphe 68a). 
51. Ibid.

52. Ibid.

53. Ibid., paragraphe $68 \mathrm{c}$ ).

54. En cela, les juges dans l'affaire Huang ont fait écho aux arguments de ceux dans l'affaire Zhang v Canada (Citizenship and Immigration), 2008 FC 533.

55. En cela, les juges dans l'affaire Huang ont fait écho aux arguments de ceux dans l'affaire Sun v Canada (Citizenship and Immigration), 2015 FC 387.

56. Article 33 de la Loi afférant à la procédure pénale, adoptée par la $2^{\text {ème }}$ Session de la 5 ème APN le premier juillet 1979, tel qu'amendée par la décision d'amender la loi afférant à la procédure pénale adoptée à la $4^{\text {ème }}$ session de la $8^{\text {ème }}$ APN, 17 mars 1996, et encore par la décision amendant la loi afférant à la procédure pénale adoptée par la $5^{\text {ème }}$ session de la $11^{\text {ème }}$ APN le 14 mars 2012.

57. Adoptée le 26 décembre 2012 par le Département de la Sécurité Publique sous $\mathrm{N}^{\circ} 127$.

58. Certes, les adeptes de sectes qualifiées de «déviantes » selon la loi pénale chinoise, telles que le Falun Gong, ou l'Eglise du Dieu Tout-Puissant, ainsi que les gestionnaires des finances de toute église sous-terraine, ou encore les fidèles qui évangélisent auprès de mineurs, sont susceptibles d'être poursuivis en application de la loi pénale.

\section{RÉSUMÉS}

Dans une série de décisions récentes, l'OFPRA et la CNDA ont fondé des rejets de demandes d'asile présentés par des chinois ayant quitté leur pays sous leur vraie identité en invoquant des rapports officiels canadiens selon lesquels les autorités chinoises interdisent aux personnes inquiétées dans les conditions alléguées par les demandeurs d'asile de quitter le territoire chinois, moyennant quoi leurs allégations de persécutions en Chine sont considérées comme dénuées de crédibilité. Dans les affaires concernées, l'OFPRA et la CNDA n'ont pas communiqué aux demandeurs d'asile lesdits rapports officiels avant de rendre leurs décisions. Cet article soutient que cette procédure viole le principe du contradictoire, que les interprétations des sources administratives canadiennes sont inexactes et que la jurisprudence canadienne, qui ne reprend pas les thèses des rapports administratifs, devrait prévaloir sur les sources administratives.

\section{AUTEUR}

\section{DANIEL ARTHUR LAPRÈS}

Avocat à la Cour d'Appel de Paris, Barrister \& Solicitor (Canada). L'auteur exprime sa reconnaissance envers Elyse Korman, Barrister \& Solicitor, Ontario, Canada, pour sa contribution en relation avec la jurisprudence canadienne. 